\title{
The epistemic integrity of scientific research
}

\author{
Jan De Winter ${ }^{\mathrm{a}}$, Laszlo Kosolosky ${ }^{\mathrm{a}}$ \\ ${ }^{a}$ Centre for Logic and Philosophy of Science, Department of Philosophy and moral sciences, Ghent University, \\ Blandijnberg 2, 9000 Ghent, Belgium
}

\begin{abstract}
We live in a world in which scientific expertise, and its epistemic authority and autonomy, become more and more important. On the other hand, the financial interests in research, which could potentially corrupt science, are increasing. Due to these two tendencies, a concern for the integrity of scientific research becomes increasingly vital. This concern is, however, hollow if we do not have a clear account of research integrity. Therefore, it is important that we explicate this concept. Following Rudolf Carnap's characterization of the task of explication, this means that we should develop a concept that is (1) similar to our common sense notion of research integrity, (2) exact, (3) fruitful, and (4) as simple as possible. Since existing concepts do not meet these four requirements, we develop a new concept in this article. We describe a concept of epistemic integrity that is based on the property of deceptiveness, and argue that this concept does meet Carnap's four requirements of explication. To illustrate and support our claims we use several examples from scientific practice, mainly from biomedical research.
\end{abstract}

Keywords: epistemic integrity; research integrity; scientific integrity; deception; biomedical research; explication

\section{Introduction}

A lot of philosophers of science, politicians and scientists, as well as the general public, consider it crucial that the integrity of scientific research is protected. Such concerns have only increased with recent large-scale shifts in science such as the commercialization and privatization of research. A writing that is especially relevant in this context, is Martinson, Anderson \& de Vries' 2005 commentary in Nature, in which a wide range of research practices that threaten the integrity of science, besides falsification, fabrication and plagiarism, is identified. Martinson, Anderson \& de Vries (2005) show that the breadth and prevalence of some of these questionable research practices is striking. This finding has intensified pre-existing worries about the integrity of scientific research. ${ }^{1}$ Given such concerns and worries, it is, however, very remarkable that it is so hard to pin down what we exactly mean by integrity (Resnik 2011, p. 399). What is exactly at

\footnotetext{
${ }^{1}$ The fact that Web of Science ${ }^{\circledR}$ currently includes 216 articles citing Martinson, Anderson \& de Vries (2005) indicates that it is a quite influential writing.
} 
stake here? What is exactly threatened when we say that something threatens the integrity of scientific research? What does it mean for scientific research to have integrity?

In this article, we explicate the concept of research integrity. Carnap characterizes the task of explication as follows:

If a concept is given as explicandum, the task consists in finding another concept as its explicatum which fulfils the following requirements to a sufficient degree.

1. The explicatum is to be similar to the explicandum in such a way that, in most cases in which the explicandum has so far been used, the explicatum can be used; however, close similarity is not required, and considerable differences are permitted.

2. The characterization of the explicatum, that is, the rules of its use (for instance, in the form of a definition), is to be given in an exact form, so as to introduce the explicatum into a wellconnected system of scientific concepts.

3. The explicatum is to be a fruitful concept, that is, useful for the formulation of many universal statements (empirical laws in the case of a nonlogical concept, logical theorems in the case of a logical concept).

4. The explicatum should be as simple as possible; this means as simple as the more important requirements (1), (2), and (3) permit. (Carnap 1950, p. 7)

Carnap states that philosophers, scientists and mathematicians make explications very frequently. Following Weber, De Vreese \& Van Bouwel (forthcoming), we wish to note, however, that the fruitfulness of philosophical explications (see third requirement) may not lie in their usefulness for formulating empirical generalizations (like scientific explications) and/or mathematical/logical theorems (like mathematical/logical explications), but rather in their usefulness for formulating clear guidelines for scientists. What we aim at in this article is then to introduce a concept, which we call "epistemic integrity," that is (1) similar to our common sense notion of research integrity, (2) exact, that is, clear and well-defined, (3) fruitful, that is, useful for the development and justification of norms for individuals and institutions involved in science, and (4) as simple as requirements (1), (2) and (3) permit.

Admittedly, several definitions of concepts like "research integrity," "integrity of the research process" and "scientific integrity" have already been offered, but these do not meet the requirements of explication to a sufficient degree. Take, for instance, Nicholas Steneck's (2006) definition. He defines research integrity as "possessing and steadfastly adhering to professional standards, as outlined by professional organizations, research institutions and, when relevant, the government and public" (Steneck 2006, p. 56). This is not very helpful to, say, a professional organization that wants to develop professional standards to which research should adhere in order to have (the highest degree of) integrity. Any standards this organization develops will guarantee research integrity according to the definition, and so the definition does not offer any guidance in outlining professional standards, which renders it useless for the organization under consideration. This means that Steneck's notion of research integrity does not meet the third requirement of explication - it is not fruitful - and therefore his account cannot be regarded as a good explication. Other accounts of research integrity (e.g., Haack 2007; Kochan 2011; National

\footnotetext{
${ }^{2}$ The reason why we call our explicatum "epistemic integrity" is that there seem to be two distinct notions of research integrity in use - an epistemic notion, which focuses on the reliability of the results of research, and a moral notion, which concerns the moral acceptability of research practices. In this article, we only focus on the epistemic notion, as this is, we believe the more common, and also the more interesting notion of research integrity.
} 
Academy of Sciences 1992; Parizeau 1999; Petrovečki \& Scheetz 2001; Office of Research Integrity 2007) have the problem that they are not sufficiently exact. They typically refer to principles such as honesty and verifiability, but do not (sufficiently) explain these principles, so that the notion of integrity remains vague.

In the next sections, we develop a concept of epistemic integrity that succeeds, as we will argue, in explicating the notion of research integrity. As our concept of epistemic integrity is based on the property of deceptiveness, we start by defining this property in the next section, and we clarify this definition on the basis of some examples from scientific practice. In section 3, we define the concept of epistemic integrity, and in section 4, we show that this concept fulfills the four requirements of explication. We conclude in section 5 .

\section{Deceptiveness}

Before we introduce our concept of epistemic integrity, we should clarify what we mean by deceptiveness, as we will refer to this property in our explication. We focus on deceptiveness of statements. When is a statement deceptive? Firstly, we should remark that whether or not a statement is deceptive depends on whom it is communicated to and his/her background assumptions. Consider a clinical trial in which part of the study population is given the most adequate doses of a new test drug, and the other part is given inadequate doses of a competing product. ${ }^{3}$ The percentage of patients that recover from a certain health condition is significantly higher in the test group. Suppose the study leader would then report that he conducted a clinical trial in which part of the study population received the test drug, and the other part received the competing product, and that a significantly higher percentage of patients recovered from their condition in the test group, without mentioning that the competing product was administered in inadequate doses. This report is deceptive - it causes people to overestimate the relative effectiveness of the test drug - but only because it is generally assumed that in active-controlled trials, all products are administered in adequate doses. People who do not make this assumption may not be misled. Those who assume that the competing product is administered in inadequate doses will not overestimate the relative effectiveness of the test drug as a result of the study leader's report, and so this report is not deceptive to them.

So the first question we have to answer is: When is a statement $s$, stated by person $P_{1}$, deceptive to a person $P_{2}$ ? We consider $s$ deceptive to $P_{2}$ if and only if (i) $s$ is presented to $P_{2}$ as a true statement, and (ii) either (a) $s$ is false, or (b) $P_{2}$ infers a false statement $s$ ' from $s$ and it is legitimate for $P_{2}$ to make this inference (given what is usually the case when a statement like $s$ is true, what persons like $P_{1}$ usually mean by statements like $s$, what $P_{1}$ says about the interpretation of $s$, etc.). $s$ is more deceptive as $P_{2}$ 's inference from $s$ to $s$ ' is more legitimate. The general degree of deceptiveness of a statement $s$ is higher as $s$ is more deceptive to a higher percentage of the people to whom it is communicated.

This account can be clarified on the basis of some examples. We start with a standard example of deception in science: a scientist, let us call him Dr. Smith, fabricates certain data, presents these data as the results of empirical investigations, and makes a certain claim, say that $\operatorname{drug} D$ does not increase the risk of heart failure $\left(s_{l}\right)$, on the basis of his so-called empirical data. Why is

\footnotetext{
${ }^{3}$ This example is not unrealistic. The commercialization of biomedical science has stimulated epistemically problematic research practices such as the one in the example (Reiss 2010, p. 432). A new test drug for a certain condition is sometimes compared to inadequate doses of some other product (Rochon et al. 1994; Tandon \& Fleischhacker 2005), or to a product that is administered in an inadequate way (Johansen \& Gøtzsche 1999).
} 
$s_{1}$ deceptive? In case drug $D$ does increase the risk of heart failure, $s_{1}$ is deceptive because it is false, while Dr. Smith presents it as true (conditions (i) and (ii)(a) are met). But even if drug $D$ does not increase the risk of heart failure, and $s_{1}$ is true, it is nevertheless highly deceptive. The reason is that people will infer from it that empirical research, namely Dr. Smith's empirical research, shows that drug $D$ does not increase the risk of heart failure $\left(s_{1}{ }^{\prime}\right)$. This inference is legitimate because Dr. Smith presents his claim as a conclusion based on data that he obtained empirically. Because the data were in fact fabricated, $s_{1}$ ' is false, and hence, $s_{1}$ is deceptive (conditions (i) and (ii)(b) are met).

Now consider a second case. Suppose a scientist states that research does not reveal whether or not climate change is occurring $\left(s_{2}\right)$, while there actually is an abundance of scientific evidence suggesting that it is. Although the scientist's statement may be true as he interprets it - e.g., research does not provide a $100 \%$ certainty about climate change - it can nevertheless be highly deceptive. This is because a lot of people will infer from $s_{2}$ that the scientific arguments for climate change do not substantially outweigh the scientific arguments against climate change $\left(s_{2}{ }^{\prime}\right)$, which is false, and this inference is legitimate because $s_{2}{ }^{\prime}$ is in fact a more obvious interpretation of $s_{2}$ than the scientist's interpretation. $s_{2}$ would be less deceptive if the scientist explained what he meant and what he didn't mean by $s_{2}$. The more this is highlighted (think of, e.g., mentioning it directly after stating $s_{2}$ versus referring to an article in which the correct interpretation is in a footnote), the less legitimate it is for the audience to keep inferring $s_{2}$ ' from $s_{2}$, and thus, the less deceptive $s_{2}$.

In the aforementioned example of the test drug being compared to inadequate doses of a competing product, the problem is similar. A lot of people will interpret the study leader's claim that the percentage of patients recovering from their health condition was significantly higher in the test group $\left(s_{3}\right)$ as follows: in the clinical trial he conducted, the percentage of patients recovering from their health condition was significantly higher in the group receiving adequate doses of the test drug than in the group receiving adequate doses of the competing product $\left(s_{3}{ }^{\prime}\right)$. Although the latter statement is false (because there was no group receiving adequate doses of the competing product), it is legitimate for the audience to infer $s_{3}$ ' from $s_{3}$, because (1) it is generally assumed that in active-controlled trials, all products are administered in adequate doses, since using inadequate doses usually does not make sense, and (2) the study leader did not mention that the competing product was administered in inadequate doses. Because conditions (i) and (ii)(b) of our account of deceptiveness are met, $s_{3}$ is deceptive (to the people inferring $s_{3}$ ' from $s_{3}$ ). To people who assume that the competing product was administered in inadequate doses, $s_{3}$ is not deceptive, since they will not infer the false statement $s_{3}$ ' from it. Note that this analysis of the deceptiveness of $s_{3}$ does not refer to any intentions. Our account implies that $s_{3}$ is deceptive to the people inferring $s_{3}$ ' from $s_{3}$, regardless of whether the study leader has the intent to deceive or not.

\section{Epistemic integrity}

Now, let us introduce our notion of epistemic integrity. While deceptiveness is, as we characterized it, a property of statements, epistemic integrity is a property of practices resulting in certain statements. More specifically, we define the epistemic integrity of a practice as a function of the degree to which the statements resulting from this practice are deceptive. The more deceptive these statements, the lower the epistemic integrity of the practice. Applied to scientific research, we get: the more deceptive the (so-called) results or conclusions of scientific research, 
the lower the epistemic integrity of this research. To illustrate this, we can again refer to the example of comparing adequate doses of a new test drug to inadequate doses of a competing product. If the report of such a study is highly deceptive (because it conceals the fact that the competing product was administered in inadequate doses), then the epistemic integrity of the research is low. So the epistemic integrity of research is determined on the basis of its results (more specifically, on the basis of how deceptive these results are).

Besides research itself, other kinds of practices in science could have a degree of epistemic integrity as well. Take, for instance, the fabrication of data which are presented as the results of empirical investigations. Since the data are fabricated, no actual research is involved. But because the practice under consideration results in a deceptive report, it could be said to have low epistemic integrity.

Finally, we should mention an important constraint on what we mean by 'statements resulting from a practice.' Here, we assume that for a statement to be considered a result of a certain practice, it should be produced within this practice, by persons or entities who are actually involved in the practice. The epistemic integrity of the practice only depends on the deceptiveness of such statements. So, for instance, if a scientist who is not involved in a certain research project draws false conclusions from it, and presents them as true, this does not entail that the research upon which he relies has low epistemic integrity. His analysis of the research and its results may have low epistemic integrity, but the epistemic integrity of the research project itself is not compromised as long as those involved in the project have not made any deceptive claims themselves.

\section{The four requirements of explication}

Is our concept of epistemic integrity a good explicatum for the concept of research integrity? In this section we examine whether it fulfils the four requirements of explication we mentioned in the introduction: similarity to the explicandum, exactness, fruitfulness and simplicity.

\subsection{Similarity to the explicandum}

The first question is whether epistemic integrity is, as we define it, sufficiently similar to our common sense notion of research integrity. As already mentioned, close similarity is not required, and considerable differences are permitted. What is required for good explication is that in most cases in which the explicandum has so far been used, the explicatum can be used (Carnap 1950, p. 7). Does our concept of epistemic integrity satisfy this requirement?

To support the claim that it does, let us take a look at a particular case in which the concept "integrity" is applied to a context of scientific research. As we have called our explicatum "epistemic integrity," we look into a case in which this specific term is used as well: Justin Biddle's 2007 essay on the Vioxx affair. The Vioxx affair concerns the questionable research on Vioxx, a painkiller owned by pharmaceutical giant Merck \& Co., Inc. In the abstract of the paper, Biddle states that "the research was organized in such a way as to allow short-term commercial interests to compromise epistemic integrity" (Biddle 2007, p. 21). Is this use of the term "epistemic integrity" compatible with our concept of epistemic integrity? Let us argue that it is.

Biddle mentions two reasons for believing that Merck's research on Vioxx was epistemically irresponsible: 
The first is that, between 2000 (or even earlier) and 2004, Merck consistently mischaracterized the current state of knowledge regarding the possible cardiovascular side effects of Vioxx. The hypothesis that Vioxx did not possess dangerous cardiovascular side effects was, until 2004, uncertain, and Merck knew that it was uncertain. Despite this, the company consistently and publicly asserted that Vioxx was safe. Secondly, there are serious questions about whether Merck honestly reported data that was unfavorable to its financial interests. Since the withdrawal of the drug, information has come to light suggesting that Merck scientists did not report all of the cardiovascular events that they should have, thus calling into question at least two of the studies that reflected favorably upon Vioxx. (Biddle 2007, p. 27)

Our account of deceptiveness implies that the two questionable research practices that this passage refers to both involve the production of deceptive claims. Firstly, consider the mischaracterization of the current state of knowledge regarding the possible cardiovascular side effects of Vioxx. An obvious implication of statements like "Merck \& Co., Inc. today reconfirmed the favorable cardiovascular safety profile of Vioxx ${ }^{\circledR}$ (rofecoxib)" (press release by Merck, entitled "Merck confirms favorable cardiovascular safety profile of Vioxx ${ }^{\circledR}$," dated May 22, 2001), is "today, Merck has sufficient scientific evidence to accept that Vioxx is safe." Therefore, it is legitimate to infer the latter from the former statement, and we can expect a lot of people to have made this inference. Because it is not true that Merck had, at the time, sufficient scientific evidence to accept that Vioxx was safe, the statement "Merck \& Co., Inc. today reconfirmed the favorable cardiovascular safety profile of Vioxx ${ }^{\circledR}$ (rofecoxib)" was highly deceptive (see our notion of deceptiveness presented in section 2 of this article). The second questionable research practice, inadequate reporting of unfavorable data, also involved the production of highly deceptive claims. When Merck scientists reported that in the VIGOR trial, 17 heart attacks occurred in the Vioxx group, while in fact there were 20, and that in the Advantage trial, five patients in the Vioxx group suffered heart attack or sudden cardiac death, while there were actually six or more, they presented false statements as true statements. Therefore, these statements were highly deceptive (again, see our notion of deceptiveness in section 2).

Because Merck's research on Vioxx resulted in highly deceptive statements, it had low epistemic integrity (see our notion of epistemic integrity presented in section 3 of this article). This could be explained by Merck's short-term commercial interests. The deceptive statements served these interests better than the truth - making the evidence for Vioxx's safety appear more compelling than it actually was, promoted the sale of Vioxx in the short term - and this may be the reason why these statements were produced. If we endorse this explanation, as we think Biddle would, then we can conclude that in the Vioxx affair, short-term commercial interests were allowed to compromise epistemic integrity, which is exactly what Biddle claims in his abstract. Thus, our concept of epistemic integrity is compatible with Biddle's use of the term.

Our concept of epistemic integrity is also compatible with Nicholas Steneck's (2006) discussion of irresponsible behavior in research. He mentions fabrication, falsification, plagiarism, misrepresentation, inaccuracy, and bias as behaviors that damage research integrity. In 4.3 of this article we show, for each of these behaviors, that our concept of epistemic integrity implies that it undermines the epistemic integrity of research. Another example is Susan Haack's (2007) account of the integrity of science. She defines it as the adherence to the values of honesty and sharing. In 4.3 of this article we show why honesty and sharing are important to protect the epistemic integrity of scientific research. There is also an overlap between the examples Haack gives of threats to scientific integrity and the examples we give in this article (e.g., she also refers 
to the Vioxx case). So her use of the terms "integrity of science" and "scientific integrity" is compatible with our concept of epistemic integrity.

We can of course not conclude on the basis of three examples (Biddle, Steneck, and Haack) that our explicatum can be used in most cases in which the explicandum has so far been used. But because exploring a larger variety of cases does not fit within the scope of this article, we leave this matter to further research. Here, we only hope to have given at least some initial plausibility to the claim that our concept of epistemic integrity fulfils the first requirement of explication.

\subsection{Exactness}

In defining our concept of epistemic integrity, we have not referred to principles such as honesty and verifiability without further specifying them. Instead, we have referred to a notion of deceptiveness that we have first specified considerably. Therefore, we consider our explicatum sufficiently clear and well-defined, and hence, we consider the second requirement of explication to be fulfilled.

\subsection{Fruitfulness}

Is our concept of epistemic integrity useful for the development and justification of norms for individuals and institutions involved in science? As such norms have already been developed in earlier work (e.g., National Academy of Sciences 2002; Resnik 1998; Shamoo \& Resnik 2009), we will not start from scratch here. Let us start from the standards for science developed in Resnik (1998). Resnik proposes the following standards:

Honesty: Scientists should not fabricate, falsify, or misrepresent data or results. They should be objective, unbiased, and truthful in all aspects of the research process.

Carefulness: Scientists should avoid errors in research, especially in presenting results. They should minimize experimental, methodological, and human errors and avoid self-deception, bias, and conflicts of interest.

Openness: Scientists should share data, results, methods, ideas, techniques, and tools. They should allow other scientists to review their work and be open to criticism and new ideas.

Freedom: Scientists should be free to conduct research on any problem or hypothesis. They should be allowed to pursue new ideas and criticize old ones.

Credit: Credit should be given where credit is due but not where it is not due.

Education: Scientists should educate prospective scientists and insure that they learn how to conduct good science. Scientists should educate and inform the public about science.

Social responsibility: Scientists should avoid causing harms to society and they should attempt to produce social benefits. Scientists should be responsible for the consequences of their research and they should inform the public about those consequences.

Legality: In the process of research, scientists should obey the laws pertaining to their work.

Opportunity: Scientists should not be unfairly denied the opportunity to use scientific resources or advance in the scientific profession.

Mutual respect: Scientists should treat colleagues with respect.

Efficiency: Scientists should use resources efficiently.

Respect for subjects: Scientists should not violate rights or dignity when using human subjects in experiments. Scientists should treat non-human, animal subjects with appropriate respect and care when using them in experiments. (Resnik 1998, chapter 4) 
What we will try to show now is that our concept of epistemic integrity is useful to justify and refine some of these standards.

Resnik uses different grounds in justifying his standards. A first is that violating the standard hinders the achievement of the goals of science. Resnik distinguishes two kinds of goals: epistemic and practical. The epistemic goals are related to the advancement of human knowledge, and the practical goals concern solving problems in engineering, medicine, economics, agriculture, and other areas of applied research (Resnik 1998, p. 39). A second kind of justification is that adhering to the standard promotes cooperation and trust in science, and a third is that it promotes the public's trust in science. Fourthly, most standards are also justified on moral grounds. We will here only focus on Resnik's epistemic justification of standards, that is, the justification of a standard on the ground that adhering to it is important for the advancement of human knowledge.

The problem with this kind of justification is that it is not clear what Resnik means by human knowledge. It is also not always clear why and how violating the relevant standards exactly impedes the advancement of human knowledge. Therefore, the claim that a certain standard is justified because violating it hinders the advancement of human knowledge, is not very informative. This is where our concept of epistemic integrity comes in. We believe that it enables us to give an alternative, more informative epistemic justification of several of Resnik's standards.

What we want is that practices in science have the highest degree of epistemic integrity, that is, we want the claims produced in science to be non-deceptive. Most of Resnik's standards for science can be justified on the basis of this desideratum. We distinguish between three kinds of standards: those that can be completely justified on the basis of this desideratum, those that can only partially be justified on the basis of this desideratum, and those that cannot be justified on the basis of this desideratum at all. The standards belonging to the latter category are: social responsibility, legality, efficiency, and respect for subjects. Adherence to these standards is important for moral reasons, not because violating them damages the epistemic integrity of scientific research.

The standards that can be completely justified on the basis of the ideal of high epistemic integrity of scientific practices are: honesty, carefulness, openness, and freedom. Firstly, take the principle of honesty: scientists should not fabricate, falsify, or misrepresent data or results, and they should be objective, unbiased, and truthful in all aspects of the research process. When scientists misrepresent data or results, e.g., when they describe a certain method of research and claim that certain data were obtained through this method while in fact they were not (e.g., because they were fabricated or falsified), they make a deceptive claim. They present a false statement ("this method delivered these data") as a true statement, and therefore it is deceptive. This means that their (so-called) research has low epistemic integrity. Since we want scientific research to have the highest degree of epistemic integrity, the conclusion is that scientists should not misrepresent data or results.

Our concept of epistemic integrity can also be used to explain why it is important that scientists are objective and unbiased in different aspects of the research process. To illustrate this, consider decisions on whether or not to publish certain results. In biomedical science, such decisions are sometimes biased; findings that are unfavorable to a certain drug (e.g., the drug is ineffective or not safe) are not published, while favorable findings are published more than once (Melander 2003; Schott et al. 2010, pp. 296-298). When this is the case, people who consult the relevant publications might infer that most research is favorable to the drug while this is in fact 
false, and this inference is legitimate because all research findings are supposed to be published not just the favorable ones. If a lot of people actually make this inference, the collection of publications can be considered highly deceptive, which means that the epistemic integrity of the body of research resulting in this collection of publications is low. So in order to guarantee that research has the highest degree of epistemic integrity, we should make sure that scientists are objective and unbiased in deciding whether or not to publish certain results.

Now consider the principle of carefulness: scientists should avoid errors in research, especially in presenting results, they should minimize experimental, methodological, and human errors, and they should avoid self-deception, bias, and conflicts of interest. Different kinds of errors could result in deceptive claims. When an instrument produces some false data (experimental error), and these data are presented as accurate, a deceptive claim is made. Deceptive claims could also be caused by methodological errors. For instance, if biomedical scientists compare adequate doses of a drug to inadequate doses of a competing product, interpret the results as saying something about the products' relative effectiveness for treating a certain condition, assuming that both are administered in their most adequate doses (methodological error), and then present this false conclusion as true, they make a deceptive claim. Finally, when scientists make a human error such as a miscalculation or inaccurate recording of data, and present the outcome as accurate, a deceptive claim is made as well.

How bias could result in deceptive claims should be clear by now. The comparison of adequate doses of a product one favors to inadequate doses of a competing product is an example of bias, and we also indicated how publication bias could lead to a highly deceptive collection of publications. Since such biases typically occur in cases in which those involved in research have conflicts of interest, we can conclude that the threat of deception is especially present in cases in which those involved in research have a conflict of interest. As errors, bias, and conflicts of interest all increase the likelihood that deceptive claims are made, a good strategy to protect the epistemic integrity of scientific research is to avoid errors, bias and conflicts of interest, which is exactly what Resnik's principle of carefulness recommends.

A third principle that can be completely justified on the basis of our concept of epistemic integrity is the principle of openness: scientists should share data, results, methods, ideas, techniques, and tools, and they should allow other scientists to review their work and be open to criticism and new ideas. Openness is important because it facilitates the detection of deceptive claims by outsiders. For example, when the use of inadequate doses of a drug in a clinical trial results in a deceptive claim, then this could easily be detected if the actual doses used are shared, but not when they are kept secret. The detection of deceptive claims by outsiders is not only valuable because this enables us to correct them or replace them by non-deceptive claims, but also because it prevents the production of deceptive claims. The easier it is for outsiders to detect deceptive claims, the more important it is for scientists to make sure that their claims are nondeceptive, since their reputation will be damaged if some of their claims are shown to be deceptive. So by making it easier for outsiders to detect deceptive claims, openness contributes to the non-deceptiveness of claims made in science, and thus, it promotes the epistemic integrity of scientific research.

Fourthly, consider the principle of freedom: scientists should be free to conduct research on any problem or hypothesis, and they should be allowed to pursue new ideas and criticize old ones. We already saw how making it easier for outsiders to detect deceptive claims promotes the epistemic integrity of research. But for outsiders to detect deceptive claims, it is not only important that information is shared with them (openness), but also that scientists are allowed to 
investigate, for any claim made, whether or not it is deceptive. Therefore, scientists' freedom to criticize claims made by others should be guaranteed.

There are also some standards that can only partially be justified on the basis of our concept of epistemic integrity: credit, education, opportunity, and mutual respect. Adherence to these standards is important regardless of whether or not violating them compromises epistemic integrity. However, some behaviors that violate them compromise epistemic integrity, and this is a reason not to practice such behaviors. Take the principle of credit: credit should be given where credit is due but not where it is not due. The main reason why this standard should be respected is that all people, including scientists, should be given just rewards for their contributions and efforts (Resnik 1998, p. 62). But some aspects of the principle of credit can also be justified on the basis of our concept of epistemic integrity. Suppose for instance that a scientist writes a text in which he presents certain ideas as his own original work, while in fact he stole these ideas from someone else (plagiarism). He then violates the principle of credit because he does not give credit where credit is due. Since the reader will infer from the text that the author has developed these ideas himself, which is not the case, the text is deceptive, which means that epistemic integrity is damaged. So for practices in science to have the highest degree of epistemic integrity, scientists should not plagiarize.

Another example is the use of guest authors and ghostwriters. In biomedical science, persons who did not make a significant contribution to a paper - typically academically affiliated investigators that are not employed by the company that financed the research - are listed as authors (guest authors) (Ross et al. 2008), while company employees who did make a significant contribution are not (ghostwriters) (Gøtzsche et al. 2007). This gives the reader a false impression of who conducted the research and who contributed what to the paper. Therefore, the statements pertaining to authorship are deceptive. So here, too, violating the principle of credit - authorship credit is not given where it is due - compromises epistemic integrity.

A second principle that can be partly justified on the basis of our concept of epistemic integrity, is the principle of education: scientists should educate prospective scientists and insure that they learn how to conduct good science, and they should educate and inform the public about science. If we want scientific research to maintain a high degree of epistemic integrity in the future, it is crucial that prospective scientists learn how to make sure that their claims are nondeceptive. They should, for instance, learn how to avoid different kinds of errors and bias, since these could all result in deceptive claims as we have seen. If scientists would not educate prospective scientists in this way, the epistemic integrity of future scientific research is threatened. Scientists should also educate and inform the public about how to interpret different scientific claims in order to avoid that people (legitimately) infer false statements from true scientific claims (which would make the scientific claims deceptive). This justification of the principle of education is, however, only partial, since prospective scientists should learn more than just how to make their claims non-deceptive.

Thirdly, we can offer a partial epistemic justification of the principle that scientists should not be unfairly denied the opportunity to use scientific resources or advance in the scientific profession (principle of opportunity). We can expect that the deceptiveness of a claim is more likely to be detected if it is investigated by people with different backgrounds, who have different perspectives on the claim. Therefore, it is important that people with different backgrounds get equal opportunities in science. As this will make it more likely that the deceptiveness of a claim is identified, we can expect more deceptive claims to be corrected or replaced by non-deceptive claims, and the incentive for scientists to make sure that their claims are non-deceptive will be stronger (as they will be more likely to get caught). This epistemic justification of the principle of 
opportunity is partial because even in a case in which unfairly denying someone the opportunity to use scientific resources or advance in the scientific profession (e.g., on the basis of his skin color) can be shown not to threaten the epistemic integrity of scientific research in any way, it is still unacceptable (on moral grounds).

A final principle we consider is the principle of mutual respect between scientists, which implies that "scientists should not harm one another, either physically or psychologically, that they should respect personal privacy, that they should not tamper with each other's experiments or results, and so forth" (Resnik 1998, p. 67). If there would not be mutual respect in science, scientists would probably be less inclined to make sure that other scientists are not deceived by the claims they make. As a consequence, we can expect more claims in science to be deceptive, and the epistemic integrity of a lot of research would be lower. Therefore, it is important that scientists adhere to the principle of mutual respect. Of course, even in cases in which one scientist can harm another without threatening epistemic integrity, scientists should not do so (on moral grounds). Therefore, our epistemic justification of the principle of mutual respect is only partial.

Finally, we wish to point out that our concept of epistemic integrity is not only useful to justify most of Resnik's standards for science, but also to refine them. Suppose a scientist fabricates certain data (e.g., in order to establish methodological or conceptual problems), that he is clear about the fact that the data are fabricated, and that there is no way in which he gives the impression that the data are not fabricated. As the principle of honesty states that scientists should not fabricate data, it implies that the scientist misbehaves. But since the scientist does not make any deceptive claim - he is clear about the fact that the data were fabricated - the epistemic integrity of his research is not undermined. Therefore, there is no reason to consider his behavior unacceptable. Data fabrication is only unacceptable when the fabricated data are presented as empirical data, that is, as the results of empirical research. The reason is that only in the latter kind of cases does data fabrication result in deceptive claims, and is the epistemic integrity of the (so-called) research undermined.

As our concept of epistemic integrity is, as we have shown, useful for the justification and refinement of standards for science, we consider it a fruitful concept. Hence, the third requirement of explication is satisfied.

\subsection{Simplicity}

In order to show that our concept of epistemic integrity is as simple as the three other requirements of explication permit, we argue that no parts of our explication can be omitted without violating the other requirements. More specifically, we argue, for each part of our definition of deceptiveness (on which our explication is based), that if it is omitted, our explicatum will be less similar to our common sense notion of research integrity. For most parts, we also argue that omitting them will make our explicatum less fruitful.

The first part we consider is condition (i) of our account of deceptiveness, which is that the deceptive statement is presented as a true statement (see section 2). Consider the following case: a scientist describes a certain hypothesis, which includes a false statement $s$, and then rejects it on the basis of empirical data. Although $s$ is false, it is not deceptive because the scientist does not present it as true; he presents it as part of an hypothesis he rejects. If condition (i) would be omitted, $s$ would be deceptive (condition (ii)(a) is met), which means that the epistemic integrity of the scientist's research would be undermined. This conflicts with our common sense notion of 
research integrity; we would not say that in the case under consideration, research integrity is threatened.

Furthermore, omitting condition (i) would also make our concept of epistemic integrity less useful for the development/justification of standards for science. It would imply that scientists should not describe hypotheses that contain false statements (otherwise epistemic integrity is undermined). This is, of course, not the kind of standard we want for science. As long as one is clear about the fact that what one is describing is only an hypothesis, which may be false, there is nothing wrong with describing an hypothesis that contains a false statement.

Secondly, consider condition (ii)(a) of our account of deceptiveness. Can we omit (ii)(a) without weakening our explication? Suppose (1) that scientists make a false claim, for instance, that scientific research unambiguously demonstrates that smoking does not increase the risk of lung cancer $\left(s_{4}\right)$, and (2) that the audience does not bother to infer anything from $s_{4}$ because it thinks the scientists cannot be trusted anyway. As the audience does not infer any false statements from $s_{4}$, condition (ii)(b) is not met. Hence, if we would omit (ii)(a) from our definition of deceptiveness, so that a claim would only be deceptive if (i) and (ii)(b) are met, $s_{4}$ would not be deceptive, and epistemic integrity would not be undermined. This conflicts with our common sense notion of research integrity; it is common sense that research integrity is damaged when scientists make false claims such as $s_{4}$, regardless of whether the audience takes these claims seriously.

Omitting (ii)(a) would also make our concept of epistemic integrity less fruitful. It would not enable us to justify the following norm: scientists should not make false claims even when the audience will not infer any false statements from these claims (e.g., because it does not take the effort to interpret these claims). Our concept of epistemic integrity, which is based on an account of deceptiveness that does include (ii)(a), does enable us to justify this norm. Even when the audience does not infer any false statements from a false claim, the claim is still deceptive according to our account of deceptiveness, as conditions (i) and (ii)(a) are met, and hence, epistemic integrity is damaged. Therefore, scientists should not make false claims, regardless of what the audience does with these claims.

Condition (ii)(b) cannot be omitted either. We have added (ii)(b) to permit that true claims can nevertheless be deceptive. Take for instance statement $s_{3}$, discussed in section 2 . We have seen that when adequate doses of a new test drug are compared to inadequate doses of a competing product in a clinical trial, and the study leader claims that the percentage of patients recovering from their health condition was significantly higher in the test group $\left(s_{3}\right)$ without mentioning that the competing product was administered in inadequate doses, then $s_{3}$ is deceptive even though it is true. Accordingly, epistemic integrity is undermined. But if (ii)(b) would be omitted, only false claims would be deceptive, and epistemic integrity would not be undermined in the case at hand. This conflicts with our common sense notion of research integrity, as we would say that research integrity is undermined in the case at hand.

Another reason why (ii)(b) cannot be omitted is that it would make our concept of epistemic integrity less fruitful. Now our concept of epistemic integrity can be used to support the norm that scientists should not engage in practices that result in deceptive claims such as $s_{3}$. If (ii)(b) would be omitted, it could not.

A final question is whether we can omit the second part of condition (ii)(b). Is it sufficient that the audience infers a false statement from a claim for this claim to be deceptive, or should this inference also be legitimate? We stated that the inference should also be legitimate because we want to avoid that the audience can turn non-deceptive claims into deceptive ones by drawing absurd conclusions from them. For instance, if the audience infers from the claim that smoking 
increases the risk of lung cancer $\left(s_{5}\right)$ that the earth is flat $\left(s_{5}\right.$ '), and deceptiveness would not require that the inference from $s$ to $s^{\prime}$ is legitimate, then this would mean that $s_{5}$ is deceptive (conditions (i) and (ii)(b) would be met), and that epistemic integrity is undermined. This conflicts with our common sense notion of research integrity, since research integrity is, according to common sense, not undermined when the audience infers $s_{5}$ ' from $s_{5}$. Because our concept of epistemic integrity does require that the inference from $s$ to $s$ ' is legitimate (which is not the case for the inference from $s_{5}$ to $s_{5}$ '), this dissimilarity with the common sense notion of research integrity is avoided.

As no parts of our account of deceptiveness, on which our concept of epistemic integrity is based, can be omitted without violating at least one of the other requirements of explication, we consider our concept of epistemic integrity as simple as possible. The fourth requirement of explication is fulfilled.

\section{Conclusion}

In this article, we tried to explicate the concept of research integrity in a way that is in accordance with Carnap's characterization of the task of explication. More specifically, we introduced a concept we called "epistemic integrity," and defined the epistemic integrity of a practice as a function of the degree to which the statements resulting from this practice are deceptive - the more deceptive these statements, the lower the epistemic integrity of the practice. Next, we argued that this concept of epistemic integrity is (1) similar to our common sense notion of research integrity, (2) exact, (3) fruitful, and (4) as simple as possible. As these are Carnap's four requirements of explication, we conclude that our concept of epistemic integrity is a good explicatum for the concept of research integrity.

We believe that our concept of epistemic integrity is also very valuable for future research. If we want to improve the quality of scientific research, we should look at epistemically problematic research practices, investigate their causes, and develop and evaluate different strategies to tackle the relevant problems. Our concept of epistemic integrity can help us in identifying epistemically problematic research practices, and it enables us to explain why these research practices are exactly epistemically problematic. Furthermore, it is also useful to assess potential solutions. We can implement different potential solutions and examine how each affects the epistemic integrity of scientific research. The more a potential solution promotes the epistemic integrity of scientific research, the better it is.

\section{Acknowledgments}

Jan De Winter is a Ph. D. fellow of the Research Foundation (FWO) - Flanders. Research for this paper by Laszlo Kosolosky was supported by subventions from the Research Foundation (FWO) - Flanders through research project G.0122.10. We are very grateful to Erik Weber for reviewing earlier versions of this paper.

\section{References}

Biddle, J. (2007). Lessons from the Vioxx debacle: What the privatization of science can teach us about social epistemology. Social Epistemology, 21(1), 21-39. 
Carnap, R. (1950). Logical foundation of probability. London: Routledge and Keegan Paul.

Gøtzsche, P. C., Hróbjartsson, A., Johansen, H. K., Haahr, M. T., Altman, D. G., \& Chan, A. (2007). Ghost authorship in industry-initiated randomised trials. PloS Medicine, 4(1), 47-52.

Haack, S. (2007). The integrity of science: What it means, why it matters. In Conselho Nacional de Ética para as Ciências da Vida, Ética e Investigação nas Ciências da Vida - Actas do $10^{\circ}$ Seminário do CNECV (pp. 9-28). Lisbon: Presidência do Conselho de Ministros. http://www.as.miami.edu/phi/haack/PORTUGAL.pdf (accessed June 4, 2012).

Johansen, H. K., \& Gøtzsche, P. C. (1999). Problems in the design and reporting of trials of antifungal agents encountered during meta-analysis. Journal of the American Medical Association, 282(18), 1752-1759.

Kochan, J. (2011). The scope and limits of scientific integrity. Paper presented at the Third Biennial Conference of the Society for the Philosophy of Science in Practice, Exeter.

Martinson, B. C., Anderson, M. S., \& de Vries, R. (2005). Scientists behaving badly. Nature, 435, 737-738.

Melander, H., Ahlqvist-Rastad, J., Meijer, G., \& Beermann, B. (2003). Evidence b(i)ased medicine - selective reporting from studies sponsored by pharmaceutical industry: Review of studies in new drug applications. BMJ, 326(7400).

National Academy of Sciences (1992). Responsible science, volume I: Ensuring the integrity of the research process. Washington, D.C.: National Academies Press.

National Academy of Sciences (2002). Integrity in scientific research: Creating an environment that promotes responsible conduct. Washington, D.C.: National Academies Press.

Office of Research Integrity (2007). Research on research integrity. http://grants.nih.gov/grants/guide/rfa-files/RFA-RR-07-004.html (accessed October 25, 2011).

Parizeau, M. (1999). Scientific integrity. In A. Montefiore, \& D. Vines (Eds.), Integrity in the public and private domains (pp. 158-171). London: Routledge.

Petrovečki, M., \& Scheetz, M. D. (2001). Croatian Medical Journal introduces culture, control, and the study of research integrity. Croatian Medical Journal, 42(1), 7-13.

Reiss, J. (2010). In favour of a Millian proposal to reform biomedical research. Synthese, 177(3), 427-447.

Resnik, D. B. (1998). The ethics of science: An introduction. London: Routledge.

Resnik, D. B. (2011). Scientific research and the public trust. Science and Engineering Ethics, 17(3), 399-409.

Rochon P. A., Gurwitz, J. H., Simms, R. W., Fortin, P. R., Felson, D. T., Minaker, K. L., \& Chalmers, T. C. (1994). A study of manufacturer-supported trials of nonsteroidal antiinflammatory drugs in the treatment of arthritis. Archives of Internal Medicine, 154(2),157163.

Ross, J. S., Hill, K. P., Egilman, D. S., \& Krumholz, H. M. (2008). Guest authorship and ghostwriting in publications related to rofecoxib. A case study of industry documents from rofecoxib litigation. Journal of the American Medical Association, 299(15), 1800-1812.

Schott, G., Pachl, H., Limbach, U., Gundert-Remy, U., Lieb, K., \& Ludwig, W. (2010). The Financing of Drug Trials by Pharmaceutical Companies and Its Consequences: Part 2: A Qualitative, Systematic Review of the Literature on Possible Influences on Authorship, Access to Trial Data, and Trial Registration and Publication. Deutsches Ärzteblatt International, 107(17), 295-301.

Shamoo, A. E., \& Resnik, D. B. (2009). Responsible conduct of research. Oxford: Oxford University Press. 
Steneck, N. H. (2006). Fostering integrity in research: Definitions, current knowledge, and future directions. Science and Engineering Ethics, 12(1), 53-74.

Tandon, R., \& Fleischhacker, W. W. (2005). Comparative efficacy of antipsychotics in the treatment of schizophrenia: A critical assessment. Schizophrenia Research, 79(2-3), 145-155.

Weber, E., De Vreese, L., \& Van Bouwel, J. (forthcoming). How to study scientific explanation? http://philsci-archive.pitt.edu/8769/1/Weber-HowTo_Study_Scientific_Explanation.pdf (accessed June 4, 2012). 